\title{
A Bidirectional Link between Brain Oscillations and Geometric Patterns
}

\author{
Federica Mauro, ${ }^{1,2,3}$ Antonino Raffone, ${ }^{3}$ and $\mathbb{C R}^{-}$ufin VanRullen ${ }^{1,2}$ \\ ${ }^{1}$ Université de Toulouse, CerCo, Université Paul Sabatier, 31062 Toulouse, France, ${ }^{2}$ CNRS, UMR 5549, Faculté de Médecine de Purpan, CHU Purpan, 31052 \\ Toulouse Cedex, France, and ${ }^{3}$ Department of Psychology, University of Rome Sapienza, 00183 Rome, Italy
}

Like hallucinogenic drugs, full-field flickering visual stimulation produces regular, geometric hallucinations such as radial or spiral patterns. Computational and theoretical models have revealed that the geometry of these hallucinations can be related to functional neuro-anatomy. However, while experimental evidence links both visual flicker and hallucinogenic drugs to upward and downward modulations of brain oscillatory activity, the exact relation between brain oscillations and geometric hallucinations remains a mystery. Here we demonstrate that, in human observers, this link is bidirectional. The same flicker frequencies that preferentially induced radial $(<10 \mathrm{~Hz})$ or spiral $(10-20 \mathrm{~Hz})$ hallucinations in a behavioral experiment involving full-field uniform flicker without any actual shape displayed, also showed selective oscillatory EEG enhancement when observers viewed a genuine static image of a radial or spiral pattern without any flicker. This bidirectional property constrains the possible neuronal events at the origin of visual hallucinations, and further suggests that brain oscillations, which are strictly temporal in nature, could nonetheless act as preferential channels for spatial information.

Key words: EEG; flicker; geometric; hallucination; oscillation

\section{Introduction}

"Form constants" are typical geometric patterns spontaneously produced by the brain under the influence of drugs (Klüver, 1928), flickering lights (Purkinje, 1819; Young et al., 1975; Becker and Elliott, 2006; Allefeld et al., 2011; Billock and Tsou, 2012), or clinical disorders such as epilepsy (Wilkinson, 2004; Billock and Tsou, 2012) and migraine (Crotogino et al., 2001; Wilkinson, 2004; Billock and Tsou, 2012). These hallucinations are often accompanied by a modulation of brain rhythmic activity (Shevelev et al., 2000; Crotogino et al., 2001; Wilkinson, 2004; Becker and Elliott, 2006; ter Meulen et al., 2009; Allefeld et al., 2011; Dubois and Vanrullen, 2011; Kometer et al., 2013; Muthukumaraswamy et al., 2013). During full-field flicker in particular, the exact class of geometric pattern experienced—radial, spiral, grid, etc.- depends on the precise flicker rate (Becker and Elliott, 2006; Allefeld et al., 2011; Elliott et al., 2012), and thus presumably on the frequency of rhythmic brain activity. While theoretical work (Ermentrout and Cowan, 1979; Bressloff et al., 2002) has established a direct correspondence between the geometrical structure of visual hallucinations and the spatial organization of visual cortex (retinotopy and cortical magnification), the contribution of brain oscillations is recognized but not fully under-

Received Jan. 29, 2015; revised April 5, 2015; accepted April 19, 2015.

Author contributions: F.M. and R.V. designed research; F.M. performed research; A.R. contributed unpublished reagents/analytic tools; F.M. and R.V. analyzed data; F.M. and R.V. wrote the paper.

This work was supported by a EURYI Award and an ERC Consolidator Grant P-CYCLES 614244 to R.V.

The authors declare no competing financial interests.

Correspondence should be addressed to Rufin VanRullen, CerCo, CNRS, Pavillon Baudot, Hopital Purpan, 31052

Toulouse Cedex (France). E-mail: rufin.vanrullen@cerco.ups-tlse.fr.

DOI:10.1523/JNEUROSCI.0390-15.2015

Copyright $\odot 2015$ the authors $\quad 0270-6474 / 15 / 357921-06 \$ 15.00 / 0$ stood (Shevelev et al., 2000; Rule et al., 2011; Billock and Tsou, 2012; Kometer et al., 2013; Muthukumaraswamy et al., 2013). To shed light on this issue, we conducted a two-part experiment testing whether the effect of oscillatory frequency on the formation of geometric visual patterns is reciprocal. We first established in a behavioral experiment (100 s uniform flickering display and no actual shape on the screen) that radial and spiral patterns are the most frequent flicker-induced hallucinations, and that radial patterns dominate at lower flicker frequencies $(<10 \mathrm{~Hz})$, whereas spirals are most probable between 10 and $20 \mathrm{~Hz}$. Subsequently, we recorded EEG while observers viewed static renditions of the radial and spiral hallucinatory patterns (12 s steady image presentation, no flicker involved). Remarkably, the same flicker frequencies that had preferentially induced radial or spiral hallucinations on a uniform flickering display (no actual shape on the screen) also showed selective oscillatory EEG enhancement when observers viewed a static radial or spiral image without flicker.

\section{Materials and Methods}

Behavioral experiment

Subjects. Eight volunteers (three females, aged 21-39, including two authors) participated in the first behavioral part of the study. All subjects had normal or corrected-to-normal visual function and no neurological disorders.

Apparatus and stimuli. Intermittent photic stimulation was delivered through a ganzfeld (i.e., a homogeneous visual field) flickering sinusoidally from black to white at various frequencies, with a small black fixation dot at the center (17 different frequencies: 3, 5, 7, 8, 9, 10, 11, 12, 13, $14,16,18,20,24,28,33$, and $40 \mathrm{~Hz}$ ). The stimulation was provided through a cathode ray monitor (resolution: $800 \times 600$; refresh rate: $160 \mathrm{~Hz}$ ) 
via the Psychophysics Toolbox (Brainard, 1997) running in MATLAB (The MathWorks).

Procedure. Participants were seated at an $\sim 60 \mathrm{~cm}$ distance from the flickering screen, with their head placed on a chin support, and instructed to fixate the black fixation dot at the center of the screen, keeping their eyes open. The screen occupied $34.1 \times 26$ degrees of visual angle.

They attended four experimental sessions, with one trial (100 s duration) per flicker frequency in each session, pseudorandomly presented $\left(4^{\star} 17=68\right.$ trials overall $)$.

At the beginning of the first experimental session, subjects were initially provided with brief descriptions and example drawings of possible hallucinatory shapes (pictures from Purkinje, 1819). They were informed that colors and motion could also be perceived (and reported), but that shape represented the variable of interest for our study. Subsequently they performed an average of three practice trials with flicker stimulation at randomly determined frequencies, openly reporting what they experienced and drawing sketches as necessary. The experimenter discussed these reports with the observer to reach a mutual consensus on their classification. This discussion procedure was also repeated at the end of each of the four experimental sessions.

During the four experimental sessions, at the end of each $100 \mathrm{~s}$ trial, subjects were required to describe the experienced hallucinatory per$\operatorname{cept}(\mathrm{s})$ in an open written report. Moreover, they were asked to indicate the experienced vividness (in a scale ranging from 0 to 10) of each reported percept; they were also encouraged to make sketches of the perceived shapes, either immediately after each trial, or at the very end of the experiment.

Data acquisition and analysis. Behavioral reports were classified by the experimenters in main pattern clusters, considering complex percept classes resulting from sketches and descriptions of the hallucinatory phenomena. The shape clusters were initially defined based on those proposed by previous studies on flicker-induced illusions (Becker and Elliott, 2006; Allefeld et al., 2011; Purkinje et al., 1819). Subsequently, definitions were refined by the experimenters, based on subjects' open reports, to restrict the number of pattern classes. Six clusters were thus identified: radial patterns, spirals, honeycombs, lines, spots, and general (other) shapes. The cluster definitions were as follows.

Radial patterns include any radial structure, or series of straight lines originating from a central point, including star and cross patternscorresponding to wheel, cross, sun, and star clusters in Allefeld et al. (2011) and radial patterns in Becker and Elliott (2006).

Spirals include any spiral structure, or curved lines around a focal point, with one or more arms, curving clockwise or counterclockwisecorresponding to spirals, ripples, and tunnels in Allefeld et al. (2011) and to spirals in Becker and Elliott (2006). Note that to limit the number of pattern classes, this definition also encompasses concentric patterns such as rings or ripples, which are sometimes treated as a separate pattern class (Allefeld et al., 2011). These patterns were very rare in our experiment, however, with only two of eight observers reporting them, and a total probability of occurrence $<2 \%$ (compared with $>48 \%$ for nonconcentric spirals).

Honeycombs are a regular arrangement of smaller geometric units pervading the screen, starting in the center, and spreading to the periphery of the visual field, possibly smaller toward the center-corresponding to honeycombs and rasters in Allefeld et al. (2011) and gratings in Becker and Elliott (2006).

Lines are stripes moving around or distributed all over the screen, zigzags, and solid, or dashed linear arrangements that could not be classified as radial or spiral patterns-corresponding to lines and bars in Allefeld et al. (2011) and lines and zigzags in Becker and Elliott (2006).

Spots include generic blobs of light emerging throughout the screencorresponding to spots in Allefeld et al. (2011) and points in Becker and Elliott (2006).

General shapes are all other percepts not classifiable in the previous categories.

The probability of occurrence of each cluster was computed for each subject and each flicker frequency. Then a global cluster probability was computed by averaging across all subjects and all frequencies of stimulation to evaluate the most frequently reported patterns. Radial and spiral clusters showed the highest probability of occurrence with, respectively, 59 and $50 \%$ of the trials, and were thus chosen for further analyses. The occurrence probability of other pattern clusters was as follows: honeycombs $19 \%$; lines $15 \%$; spots $2 \%$; general shapes $19 \%$. Occurrence distributions for each subject and each pattern were fitted to Weibull functions (nonlinear least-squares fit), and the peak frequencies of the radial versus spiral distribution fits were compared across subjects with a paired two-tailed $t$ test. To estimate the frequencies at which the probabilities of reporting radial versus spiral hallucinations were most different (which may not be identical to the peak frequency of each pattern), for each subject we computed the difference between the two distribution fits (spiral-radial); the minimum and maximum of this function (within the range $2-25 \mathrm{~Hz}$ ) gave us the frequencies at which radial and spiral patterns (respectively) were more likely to occur than the other pattern. We compared these frequencies across subjects with a paired twotailed $t$ test.

For the purpose of the second part of the study, eight prototypical static stimuli for radial and spiral patterns were created based on subjects' reports and sketches, varying certain shape characteristics such as radius, phase, and number of radial spokes or spiral arms. All shapes were produced as different transparent apertures of a gray uniform foreground into the same textured background. The resulting geometric patterns were then presented to the participants who rated their resemblance to actually experienced hallucinatory patterns on a Likert scale (ranging from 0 , not at all to 3, completely resembling).

Two prototypical pictures were chosen, one radial and one spiral, which scored the highest average value on the Likert scale (1.6 and 2.1 for the radial and spiral pictures, respectively). The chosen images were then equalized for luminance, contrast, and 2D Fourier power spectrum.

\section{Electrophysiological experiment}

Participants. Twenty volunteers participated in the second electrophysiological experiment (11 female, aged 21-36, including one author). All subjects had normal or corrected-to-normal visual function and no neurological disorders.

Apparatus and stimuli. EEG and EOG were recorded at $1024 \mathrm{~Hz}$ using an ActiveTwo BioSemi system (64 cranial and three ocular active electrodes), while stimulus presentation was delivered through a cathode ray monitor (resolution: $1024 \times 768$; refresh rate: $100 \mathrm{~Hz}$ ) via the Psychophysics Toolbox (Brainard, 1997) running in MATLAB (The MathWorks).

Procedure. Participants were sitting at an $\sim 60 \mathrm{~cm}$ distance from the screen, with their head placed on a chin support, and instructed to fixate the black fixation dot in the center of the screen. They attended one experimental session during which the two static pictures were pseudorandomly presented, 120 times each in $12 \mathrm{~s}$ trials. EEG and EOG recordings were acquired concurrently. The pictures subtended $36.5 \times 27$ degrees of visual angle.

Data acquisition and analysis. EEG recordings were downsampled offline to $256 \mathrm{~Hz}$ for data analysis via the EEGLAB (Delorme and Makeig, 2004) toolbox for MATLAB. Data were re-referenced to average reference, notch-filtered (band-stop 45-55 Hz) and high-pass filtered (cutoff $0.5 \mathrm{~Hz}$ ), and then epoched from $1000 \mathrm{~ms}$ before to $12,000 \mathrm{~ms}$ after stimulus onset. Baseline EEG activity from the prestimulus interval $(-1000 \mathrm{~ms})$ was subtracted from each trial. Finally, all trials were visually inspected for artifacts and eye movements, and potentially rejected; channels containing abundant artifacts were discarded and replaced by an interpolation of adjacent electrodes.

Grand-average ERPs (mean over trials) to radial and spiral trials were compared across subjects using a paired $t$ test (19 degrees of freedom, $p<$ 0.05 , FDR-corrected for multiple comparisons across electrodes and time points)

EEG spectral analyses were based on a single-trial time-frequency transform from the EEGLAB (Delorme and Makeig, 2004) toolbox, akin to a wavelet transform with three cycles at $4 \mathrm{~Hz}$ and increasing linearly to 15 cycles at $45 \mathrm{~Hz}$. For each subject, the amplitudes of wavelet coefficients were averaged over time, trials, and electrodes for each frequency. The resulting amplitude spectra, expressed in decibels, were compared statis- 


\section{A Flicker-induced hallucinations}
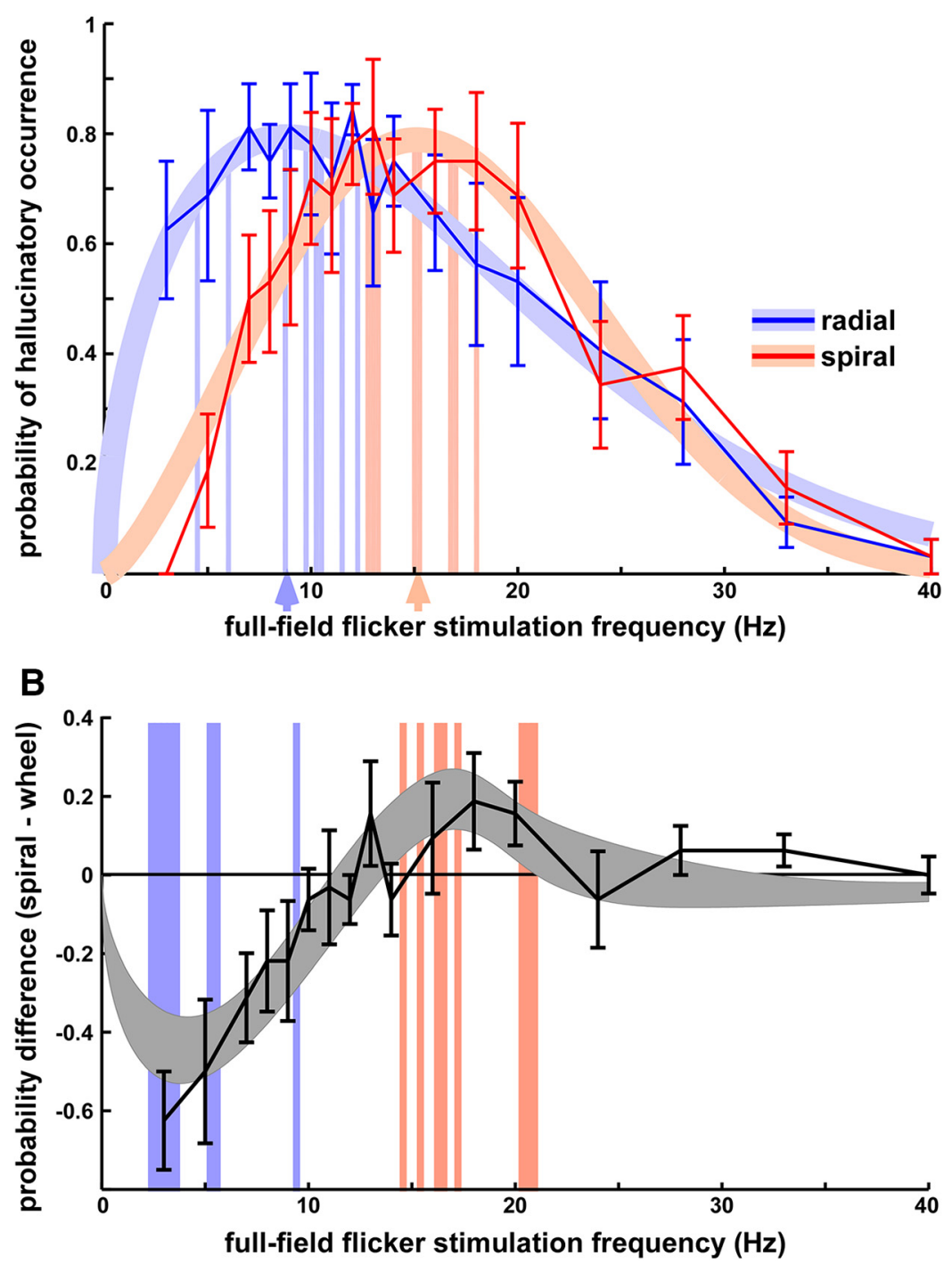

Figure 1. Radial and spiral hallucinatory patterns are preferentially elicited by flicker at distinct frequencies. $A$, Probability of occurrence of each hallucinatory pattern in relation to flicker frequency. Error bars represent SEM across observers $(N=8)$. Thick background lines represent the best-fitting Weibull function to the grand-average data, with vertical arrows pointing to the corresponding peak frequency on the $x$-axis. Each colored vertical line in the background denotes the peak frequency for reporting a radial (blue line) or a spiral pattern (red line) of an individual subject (line thickness indicates the number of subjects with the same peak frequency). The difference in peak frequency was statistically significant across observers (paired $t$ test, $t_{(7)}=9.5, p<$ $0.00003)$. $\boldsymbol{B}$, Difference between report probabilities of the two shapes (black line; error bars represent SEM across observers). The shaded gray area indicates the mean difference ( \pm SEM across observers) between Weibull-function fits of individual subjects. The minimum and maximum frequencies for this difference are 4 and $17 \mathrm{~Hz}$, respectively. Each colored vertical line in the background denotes the minimum (blue line) and maximum (red line) frequencies of an individual subject (line thickness indicates the number of subjects with the same frequency).

tically across subjects using a paired $t$ test (19 degrees of freedom, $p<$ 0.05 , FDR-corrected for multiple comparisons across frequencies).

\section{Results}

A first group of eight observers was instructed to report any occurrence of a geometric visual hallucination experienced during full-field flicker at a frequency ranging from 3 to $40 \mathrm{~Hz}$ (17 distinct frequencies, $100 \mathrm{~s}$ trials repeated four times for each frequency in randomized order, and free written report at the end of each trial). The reports highlighted two classes of patterns that were consistently perceived by all subjects: radial and spiral patterns. For each of these two patterns, the probability of occur- rence strongly varied depending on flicker frequency, with a maximum at intermediate frequencies $\sim 10 \mathrm{~Hz}$, as previously reported (Shevelev et al., 2000; Becker and Elliott, 2006; Allefeld et al., 2011). Importantly, this maximum frequency differed systematically for the two patterns (Fig. 1): overall, radial-like hallucinations were most likely to occur during flicker at 8.8 $\mathrm{Hz}$ and spiral-like hallucinations at 15.1 $\mathrm{Hz}$; across individual observers, the maximal frequencies of $9.2 \pm 2.7$ and $15.1 \pm$ $2.0 \mathrm{~Hz}$, respectively, for radial and spiral patterns (mean \pm SD across observers) were statistically different (paired $t$ test, $\left.t_{(7)}=9.5, p<0.00003\right)$. Relative to one another, radial hallucinations were more likely to occur at $\sim 4.4 \pm 2.2 \mathrm{~Hz}$ (mean \pm SD across observers) and spirals at $\sim 17.7 \pm 2.5 \mathrm{~Hz}$ (Fig. $1 B$ ). Again, the frequency difference was highly significant across observers $\left(t_{(7)}=11.3, p<10^{-5}\right)$. In conclusion, the first part of the study revealed that different geometric hallucinations are produced by full-field flicker at different optimal frequencies.

Next, we asked whether the relation between flicker frequency and geometric shape could be reversed: do actual (rather than illusory) radial and spiral images induce different brain oscillatory responses? Based on subjective reports and drawings previously made by the first group of eight observers, two static prototypical pictures of the illusory patterns (one radial and one spiral) were created and subsequently validated by the observers. The two resulting stimuli were equalized for luminance, contrast, and 2D Fourier power spectrum (Fig. 2A) and used in the second part of the study, aimed at investigating the oscillatory brain activity associated with their perception. A distinct group of subjects $(N=20)$ undergoing EEG and EOG recordings passively viewed each picture 120 times (in randomized order), statically displayed at the center of the screen for $12 \mathrm{~s}$. During each trial they were simply instructed to fixate a black point at the center of the screen. Trials with eye movements visible in EOG traces were rejected from analysis.

The ERPs elicited in response to stimulus onset were compared statistically between the two patterns (Fig. $2 B$ ). The first statistical difference (paired $t$ test, 19 degrees of freedom, $p<0.05$ FDR-corrected for multiple comparisons across electrodes and time points) appeared $135 \mathrm{~ms}$ after stimulus onset over occipitotemporal electrodes, most prominently on the right side. Importantly, no early occipital differences were visible, suggesting that the two stimuli activate early visual cortex to the same extent (as expected due to our image equalization procedure). Instead, the occipitotemporal difference peaking at $\sim 170 \mathrm{~ms}$ is compatible with activity recorded from shape-selective intermediate visual 
areas (Gallant et al., 1993; Allison et al., 1999). We thus selected for further analysis the two occipitotemporal electrodes (P8 and PO8) with maximal differential activity.

For each trial, EEG data from selected electrodes were subjected to a waveletbased time-frequency transform. The amplitude of wavelet coefficients was averaged over trials, time points, and electrodes, separately for the two geometric shapes. The resulting amplitude spectra were compared statistically (Fig. 2C). Systematic spectral differences were visible $(p<0.02$, FDR-corrected for multiple comparisons across frequencies), such that lower frequencies $(4-6 \mathrm{~Hz})$ were relatively enhanced by viewing the radial pattern, and higher frequencies (11-21 $\mathrm{Hz}$ ) by the spiral shape. This pattern of EEG spectral differences during static viewing of real shapes is strikingly similar to the difference in behavioral reports of illusory shapes induced by flickering stimulation (compare Figs. 1B, 2C). We also verified that the spectral differences were not indirectly caused by systematic variations in eye movements: no significant spectral difference was found when performing the same spectral analysis on horizontal and vertical EOG channels.

\section{Discussion}

A number of previous studies have investigated flicker-induced hallucinations (ter Meulen et al., 2009). In particular, Becker and Elliott (2006) and Allefeld et al. (2011) varied flicker frequency in a systematic way and measured concurrent changes in the probability of perceiving different geometric hallucinations, as done in our behavioral experiment. Although the various studies are difficult to compare because of several factors (specifically: different definitions of pattern clusters, different ranges of flicker frequencies investigated, use of CRT screen flicker in our study vs LED flicker in past studies, use of sine-wave flicker in our study vs square-wave in past studies, longer stimulation durations of $100 \mathrm{~s}$ at fixed flicker frequency in our study vs $60 \mathrm{~s}$ and $30 \mathrm{~s}$ in Becker and Elliott, 2006 and continuous frequency sweeping at $0.1 \mathrm{~Hz} / \mathrm{s}$ in Allefeld et al., 2011), it is worth noting that all studies described relatively high probability of occurrence for radial and spiral patterns (Becker and Elliott, 2006 measured an occurrence probability of 27 and $22 \%$ for radial and spiral patterns, respectively). Importantly, the two classes of patterns were optimally elicited at different flicker frequencies (12 and $17 \mathrm{~Hz}$ for

\section{A Static stimulus presentation (12s trials)}
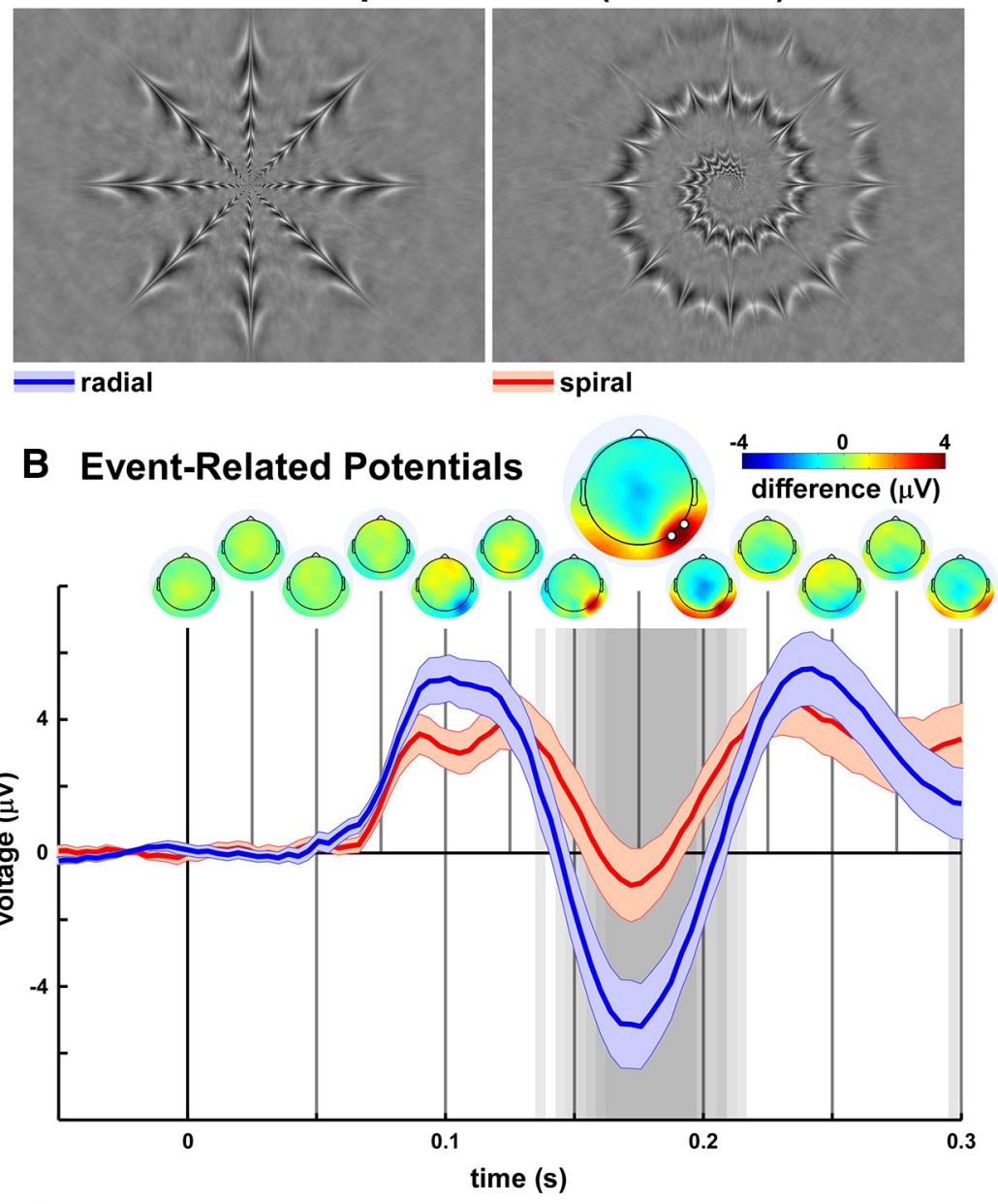

\section{Spectral differences}

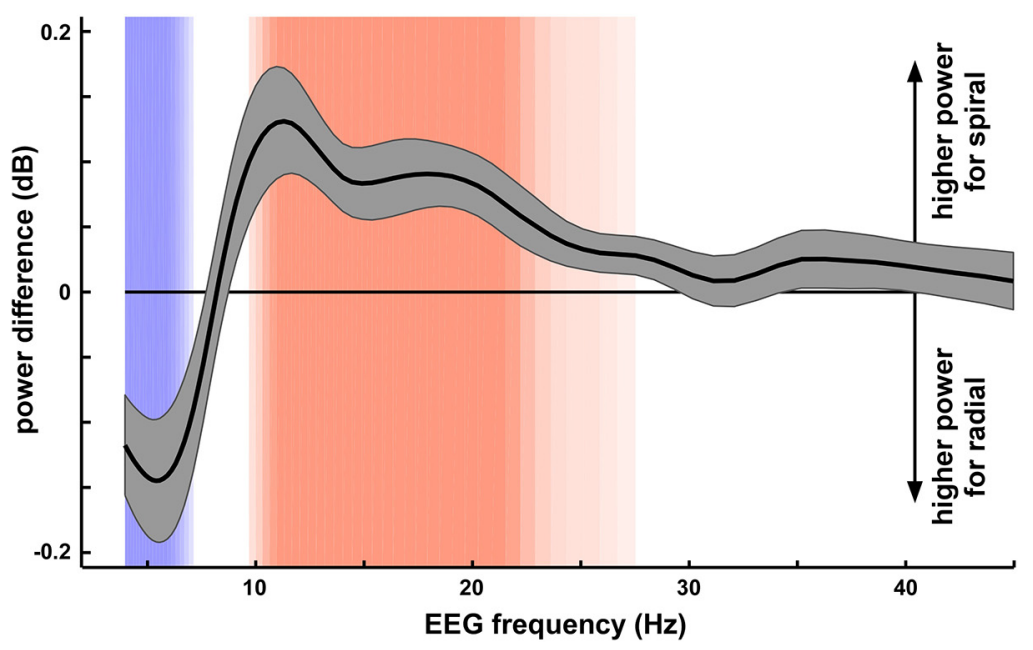

Figure 2. Radial and spiral images preferentially enhance distinct brain oscillatory frequencies. $A$, Facsimile versions of the radial and spiral hallucinatory patterns. These two images, equalized for low-level properties, were presented statically for $12 \mathrm{~s}$ while EEG responses were recorded. $\boldsymbol{B}$, ERPs to trial onset revealed that differential activity between radial and spiral images was restricted to occipitotemporal sites (scalp-topography time line on top) and at relatively long latencies (peaking at $\sim 170 \mathrm{~ms}$ ). The background grayscale indicates significance of a paired $t$ test across observers $(N=20 ; p<0.05$, FDR-corrected for multiple comparisons across electrodes and time points). The enlarged scalp map illustrates the topography at the time of maximal difference. The two highlighted points on this map mark the electrodes on which the ERP displayed underneath was computed, and which were selected for further analysis. C, Spectral power differences between EEG signals recorded during static viewing of radial versus spiral images. The background colors indicate significance of a paired $t$ test across observers ( $p<0.05$, FDR-corrected; strongest color for $p<0.02$, FDR-corrected), with blue denoting frequencies of higher power for radial images (peaking between 4 and $6 \mathrm{~Hz}$ ) and red higher power for spiral images (peaking between 11 and $21 \mathrm{~Hz}$ ). 
radial and spiral patterns, respectively, in Becker and Elliott, 2006 ) that match reasonably well the ones reported here ( $\sim 9$ and $15 \mathrm{~Hz}$; Fig. 1A). Therefore, despite the abovementioned differences, our behavioral findings are globally consistent with past observations of frequency-dependent geometric hallucinations. Furthermore, we show here for the first time that this frequency dependence can be reversed, i.e., that the relation between oscillatory frequency and geometric shape perception is bidirectional.

A recently discovered illusion called the "flickering wheel" revealed that a static radial geometric pattern (a wheel with 32 spokes) could induce a resonance with a specific brain rhythm (in the alpha range, $8-12 \mathrm{~Hz}$ ), which could be directly experienced as an illusory flicker (Sokoliuk and VanRullen, 2013). The present study implies that this "geometric resonance" could be a more general phenomenon, with different shapes amplifying different frequencies (though not always above the threshold of flicker perception: none of the subjects in the EEG experiment reported perceiving flicker within the static radial or spiral pattern). Most importantly, it demonstrates that the association is bidirectional, such that activation of the specific frequency through flicker can also produce an illusory image of the corresponding shape. The observed bidirectional link between geometric patterns and brain rhythms can inform us about the processing of visual shape and the role of oscillations in the human brain. What neural mechanisms could underlie such a bidirectional relation?

One possibility follows from an influential model of pattern formation (Rule et al., 2011) in which periodic stimulation (i.e., flicker) interacts with lateral inhibition mechanisms in primary visual cortex to create geometric activation patterns. In a certain range of stimulation frequencies (which depends on neuronal time constants and other parameters), the model produces parallel cortical stripes; in turn, horizontal and diagonal stripes in cortex, when translated to visual-world coordinates, correspond, respectively, to perceived radial and spiral shapes (Ermentrout and Cowan, 1979; Bressloff et al., 2002). In the context of this model, the present behavioral findings (Fig. 1) would imply that flicker frequency influences the orientation of activity stripes in cortex. In counterpart, the present EEG results (Fig. 2) suggest that cortical stripe orientation could directly affect the frequency of brain rhythms in the $4-20 \mathrm{~Hz}$ range.

A second possibility is rooted in recent experimental work revealing a negative gradient of optimal stimulation frequencies from primary to higher level visual areas (McKeeff et al., 2007; Gauthier et al., 2012; Rossion, 2014); for example, the optimal stimulus to activate face-selective higher visual areas is a face flickering at low frequencies $(\sim 5 \mathrm{~Hz})$, whereas $\mathrm{V} 1$ will be most activated at higher frequencies $(\sim 20 \mathrm{~Hz})$. Assuming that radial and spiral shapes are preferentially processed in two distinct cortical regions or subregions with two different optimal stimulation frequencies, it becomes conceivable that visual flicker around one or the other frequency, in the absence of any actual shape input, could be sufficient to elicit an illusory image of the corresponding pattern-radial or spiral-depending on the frequency and hence on the activated region (Fig. 1). One might even imagine that the abovementioned pattern formation mechanisms in V1 might be frequency independent but instrumental in providing, through feedback circuits, the missing shape input to frequencyselective higher brain regions (Billock and Tsou, 2007, 2010). This idea is compatible with recent experimental and theoretical observations suggesting that geometric hallucinations could originate, at least in part, in higher visual brain regions (Ffytche, 2008; Kometer et al., 2011, 2013; Froese et al., 2013). It is also consistent with the topography of the EEG effects (Fig. 2), highlighting occipitotemporal regions known to support higher level visual processes. To complete the explanation, one would also need to assume that a static presentation of the visual pattern preferentially processed by one or the other brain region would initiate rhythmic activity in the corresponding region at the corresponding frequency (Fig. 2). This would amount to a system in which those brain regions (and possibly all of visual cortex) work as an FM radio, with specific frequencies in the $4-20 \mathrm{~Hz}$ range carrying different information content from different "stations."

\section{References}

Allefeld C, Pütz P, Kastner K, Wackermann J (2011) Flicker-light induced visual phenomena: frequency dependence and specificity of whole percepts and percept features. Conscious Cogn 20:1344-1362. CrossRef Medline

Allison T, Puce A, Spencer DD, McCarthy G (1999) Electrophysiological studies of human face perception. I: Potentials generated in occipitotemporal cortex by face and non-face stimuli. Cereb Cortex 9:415-430. CrossRef Medline

Becker C, Elliott MA (2006) Flicker-induced color and form: interdependencies and relation to stimulation frequency and phase. Conscious Cogn 15:175-196. CrossRef Medline

Billock VA, Tsou BH (2007) Neural interactions between flicker-induced self-organized visual hallucinations and physical stimuli. Proc Natl Acad Sci U S A 104:8490-8495. CrossRef Medline

Billock VA, Tsou BH (2010) Seeing forbidden colors. Sci Am 302:72-77. CrossRef Medline

Billock VA, Tsou BH (2012) Elementary visual hallucinations and their relationships to neural pattern-forming mechanisms. Psychol Bull 138: 744-774. CrossRef Medline

Brainard DH (1997) The Psychophysics Toolbox. Spat Vis 10:433-436. CrossRef Medline

Bressloff PC, Cowan JD, Golubitsky M, Thomas PJ, Wiener MC (2002) What geometric visual hallucinations tell us about the visual cortex. Neural Comput 14:473-491. CrossRef Medline

Crotogino J, Feindel A, Wilkinson F (2001) Perceived scintillation rate of migraine aura. Headache 41:40-48. CrossRef Medline

Delorme A, Makeig S (2004) EEGLAB: an open source toolbox for analysis of single-trial EEG dynamics including independent component analysis. J Neurosci Methods 134:9-21. CrossRef Medline

Dubois J, Vanrullen R (2011) Visual trails: do the doors of perception open periodically? PLoS Biol 9:e1001056. CrossRef Medline

Elliott MA, Twomey D, Glennon M (2012) The dynamics of visual experience, an EEG study of subjective pattern formation. PLoS One 7:e30830. CrossRef Medline

Ermentrout GB, Cowan JD (1979) A mathematical theory of visual hallucination patterns. Biol Cybern 34:137-150. CrossRef Medline

Ffytche DH (2008) The hodology of hallucinations. Cortex 44:1067-1083. CrossRef Medline

Froese T, Woodward A, Ikegami T (2013) Turing instabilities in biology, culture, and consciousness? On the enactive origins of symbolic material culture. Adaptive Behav 21:199-214. CrossRef

Gallant JL, Braun J, Van Essen DC (1993) Selectivity for polar, hyperbolic, and Cartesian gratings in macaque visual cortex. Science 259:100-103. CrossRef Medline

Gauthier B, Eger E, Hesselmann G, Giraud AL, Kleinschmidt A (2012) Temporal tuning properties along the human ventral visual stream. J Neurosci 32:14433-14441. CrossRef Medline

Klüver H (1928) Mescal: the divine plant and its psychological effects. London: Kegan Paul.

Kometer M, Cahn BR, Andel D, Carter OL, Vollenweider FX (2011) The 5-HT2A/1A agonist psilocybin disrupts modal object completion associated with visual hallucinations. Biol Psychiatry 69:399-406. CrossRef Medline

Kometer M, Schmidt A, Jäncke L, Vollenweider FX (2013) Activation of serotonin $2 \mathrm{~A}$ receptors underlies the psilocybin-induced effects on alpha oscillations, N170 visual-evoked potentials, and visual hallucinations. J Neurosci 33:10544-10551. CrossRef Medline

McKeeff TJ, Remus DA, Tong F (2007) Temporal limitations in object processing across the human ventral visual pathway. J Neurophysiol 98:382393. CrossRef Medline 
Muthukumaraswamy SD, Carhart-Harris RL, Moran RJ, Brookes MJ, Williams TM, Errtizoe D, Sessa B, Papadopoulos A, Bolstridge M, Singh KD, Feilding A, Friston KJ, Nutt DJ (2013) Broadband cortical desynchronization underlies the human psychedelic state. J Neurosci 33:15171-15183. CrossRef Medline

Purkinje JE (1819) Beobachtungen und versuche zur Phisologie der Sinne: Beiträge zur Kenntniss des Sehens in Subjectiver Hinsicht. Prague: Calve.

Rossion B (2014) Understanding individual face discrimination by means of fast periodic visual stimulation. Exp Brain Res 232:1599-1621. CrossRef Medline

Rule M, Stoffregen M, Ermentrout B (2011) A model for the origin and properties of flicker-induced geometric phosphenes. PLoS Comput Biol 7:e1002158. CrossRef Medline
Shevelev IA, Kamenkovich VM, Bark ED, Verkhlutov VM, Sharaev GA, Mikhailova ES (2000) Visual illusions and travelling alpha waves produced by flicker at alpha frequency. Int J Psychophysiol 39:9-20. CrossRef Medline

Sokoliuk R, VanRullen R (2013) The flickering wheel illusion: when alpha rhythms make a static wheel flicker. J Neurosci 33:13498-13504. CrossRef Medline

ter Meulen BC, Tavy D, Jacobs BC (2009) From stroboscope to dream machine: a history of flicker-induced hallucinations. Eur Neurol 62:316320. CrossRef Medline

Wilkinson F (2004) Auras and other hallucinations: windows on the visual brain. Prog Brain Res 144:305-320. CrossRef Medline

Young RS, Cole RE, Gamble M, Rayner MD (1975) Subjective patterns elicited by light flicker. Vision Res 15:1291-1293. CrossRef Medline 